\title{
Análisis de las políticas de I+D+i en Panamá implementadas por SENACYT
}

\author{
Jorge Caldera-Serrano \\ Universidad de Extremadura, Facultad de Ciencias de la Documentación y la Comunicación, Badajoz, \\ España \\ jcalser@unex.es
}

DOI: https://doi.org/10.26512/rici.v12.n3.2019.24441

Recebido/Recibido/Received: 2019-05-01

Aceitado/Aceptado/Accepted: 2019-08-21

ARTIGOS DE REVISÃO

Resumen: Se analizan las políticas que en materia de I+D+i se desarrollan desde La Secretaría Nacional de Ciencia, Tecnología e Innovación (SENACYT), organismo autónomo con dependencia económica del Gobierno de la República de Panamá. El objetivo es mostrar la situación actual, logros así como los principales puntos débiles o mejoras que desde el Gobierno de Panamá se debe afrontar con urgencia para implantar un sistema nacional de investigación sostenible, de calidad y con mayor impacto y visibilidad. Para ello, se han analizado las propias memorias, proyectos y planes publicados por SENACYT, con el fin de extraer la información de la principal fuente de información. Una vez analizado, se ha detectado las principales carencias siendo plasmada en elementos de mejora, en unos casos, o acciones inexistentes que deben abordarse.

Palabras claves: Política científica. Ciencia. Tecnologia. Panamá. Sistema Nacional de Ciencia.

\section{Analysis for policies in Research, Development and Innovation in Panama implemented by SENACYT}

Abstract: It analyzes the policies that in science, technology and innovation are developed from the National Secretariat of Science, Technology and Innovation (SENACYT) - autonomous organization - with economic dependence of the Government of the Republic of Panama. The objective is to show the current situation, achievements as well as the main weak points or improvements that the Government of Panama must face urgently to implement a national system of sustainable research, quality and greater impact and visibility. For this purpose, the reports, projects and plans published by SENACYT have been analyzed, in order to extract the information from the main source of information. Once analyzed, the main deficiencies have been detected, being reflected in the improvement element, in some cases, or nonexistent actions that must be addressed.

Keyword: Scientific policies. Science. Technology. Panamá. National System of Science.

\section{Análise das políticas de P \& D \& I no Panamá implementadas pelo SENACYT}

Resumo: Analisa as políticas que em ciência, tecnologia e inovação são desenvolvidas a partir da Secretaria Nacional de Ciência, Tecnologia e Inovação (SENACYT) - organização autônoma - com dependência econômica do Governo da República do Panamá. O objetivo é mostrar a situação atual, as conquistas, bem como os principais pontos fracos ou melhorias que o Governo do Panamá deve enfrentar com urgência para implementar um sistema nacional de pesquisa sustentável, qualidade e maior impacto e visibilidade. Para tanto, foram analisados os relatórios, projetos e planos publicados pelo SENACYT, a fim de extrair as informações da principal fonte de informação. Uma vez analisadas, as principais 
deficiências foram detectadas, refletindo-se no elemento de melhoria, em alguns casos, ou em ações inexistentes que devem ser abordadas.

Palavras-chave: Panamá. Política científica. Ciência. Tecnologia. Sistema Nacional de Ciência.

\section{Introducción}

República de Panamá. País ubicado en América Central. Fronterizo con Colombia y Costa Rica, y con El Caribe y el Pacífico. Con una población de algo más de cuatro millones de habitantes. Con un PIB de 105.000 millones de dólares, que lo coloca en el puesto 85 del mundo por PIB (Wikipedia, 2018).

Según el Foro Económico Mundial es el segundo país más competitivo de América Latina y el país con mayor crecimiento económico (Mundo Social, 2017).

Entre la larga y complicada Historia de Panamá desde la independencia de España el 28 de noviembre de 1821 y su unión y separación a diferentes países o agrupación de países, un momento clave de despegue para este país ha sido la recuperación del Canal de Panamá desde el 31 de diciembre de 1999 (el 75\% de la economía panameña se centra en los servicios financieros, turísticos y logísticos).

País inmensamente atractivo para la inversión por sus beneficios y exenciones fiscales, y fascinante por su belleza, no cuenta con una estructura eficiente en materia de Ciencia, Tecnología e Innovación derivado de la falta de políticas científicas decididas y proactivas que pongan a la investigación como eje vertebrador del desarrollo industrial, logístico, turístico, educativo, etc. del desarrollo del país.

Aunque se han realizado grandes e importantes esfuerzos, especialmente con la implementación y desarrollo de la Secretaría Nacional de Ciencia, Tecnología e Innovación, aún son muchos los retos y potenciales acciones que pueden desarrollarse para mejorar a la investigación como eje estratégico del desarrollo del país. Y todo ello poniendo al sistema educativo -especialmente al universitario- al frente del proceso transformador por medio del desarrollo de una cultura de I+D+i.

El trabajo que exponemos es un análisis de las políticas implementadas por la SENACYT al amparo de los diferentes planes estratégicos nacionales, y muy especialmente al momento actual. Con este trabajo se quiere dotar de un estudio que sirva para la toma de decisiones en materia de política científica, derivado de que se incide dónde y en qué apartado se debe mejorar y también cuáles son aquellos elementos en los que se debe comenzar a trabajar de una manera más tajante.

La estabilidad económica y política del país no está siendo transmitida al sistema de investigación y desarrollo, y apostamos por una mejora en la gobernanza, afianzamiento por ley de la financiación, y estabilidad en las políticas. El potencial de toda la región, en este caso de la 
República de Panamá, lo hace merecedor de un mejor y más potente sistema nacional de investigación que haga posible que la ciencia panameña comience a posicionarse a nivel internacionales, y que sus trabajos tengan una corresponsabilidad con la ciudadanía panameña.

\section{La Secretaría Nacional de Ciencia, Tecnología e Innovación (Panamá)}

La Secretaría Nacional de Ciencia, Tecnología e Innovación (SENACYT) es la institución pública panameña dependiente de la República de Panamá (institucionalmente autónoma, en su estructura organizacional) creada en el año 1992 como organismo adjunto a la Presidencia de la República de Panamá, y modificada legislativamente con posterioridad en los años 2005 y 2007, en la que confiere la autonomía institucional, con el objetivo de "convertir a la ciencia, la tecnología la innovación en instrumentos de desarrollo el país" (Crespi, et al., 2011). SENACYT trabaja atendiendo a las líneas marcadas en el Plan Estratégico Nacional de Ciencia, Tecnología

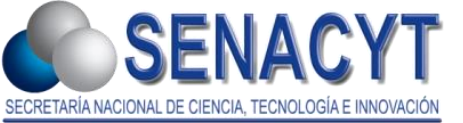
e Innovación (PENCYT) para los años 2015-2019 (Panamá, 2015), por el Gobierno de Panamá, de ahí que la independencia institucional queda relegada al seguir los planes de PENCYT $y$, evidentemente, por la dependencia económica. Por lo tanto, dicha independencia instrumental y organizacional parece oportuna para garantizar la independencia a la hora de gestionar el dinero público, lo que no quita que dicha independencia no deje sin aporte económico suficiente e infraestructura necesaria para la consecución de los fines de la SENACYT.

Tal y como especifica la web de la SENACYT (http://www.senacyt.gob.pa/)-fuente principal para extraer la información de este apartado- las diferentes actuaciones ya sea por medio de actividades, proyectos así como el desarrollo de los programas desarrollados por la SENACYT tienen la finalidad de fortalecer y desarrollar la ciencia, la tecnología y la innovación en Panamá, con el fin de aumentar la productividad y la competitividad tanto en el sector privado, al gobierno, a la universidad a la ciudadanía en general.

Tanto la Misión como la Visión aportada por la SENACYT va encaminada a convertirse y constituirse como el motor de desarrollo de la ciencia, la tecnología y la innovación de la sociedad panameña, por medio de la implementación y puesta en marcha de la política nacional de ciencia e investigación.

Para terminar de presentar el interés y la importancia de SENACYT en y para Panamá, identificamos los valores con los que cuenta: a) creatividad, como método para la solución de los problemas sociales; b) excelencia, como método de trabajo interno y como mejor manera de motivar la mejor ciencia; c) relevancia, para cambiar la realidad panameña; d) transparencia, como principio con los actores del sistema y con sus actuaciones; e) solidaridad, como responsable ante la sociedad del liderazgo nacional (SENACYT, 2018). 
La Junta Directiva de la institución está compuesta por altas personalidades de Panamá, tales como:

- Ministro de la Presidencia y presidente de la Junta Directiva de la SENACYT.

- Viceministro Académico del Ministerio de Educación (designado por el Ministerio de Educación).

- Representante de la Asociación Bancaria de Panamá.

- Representante del Consejo Nacional de la Empresa Privada.

- Representante de los Centros de Investigación No Gubernamentales.

- Representante de los Centros de Investigación del Sector Público.

- Representante del Consejo de Rectores de Panamá.

- Imagen 1. Organigrama de la SENACYT

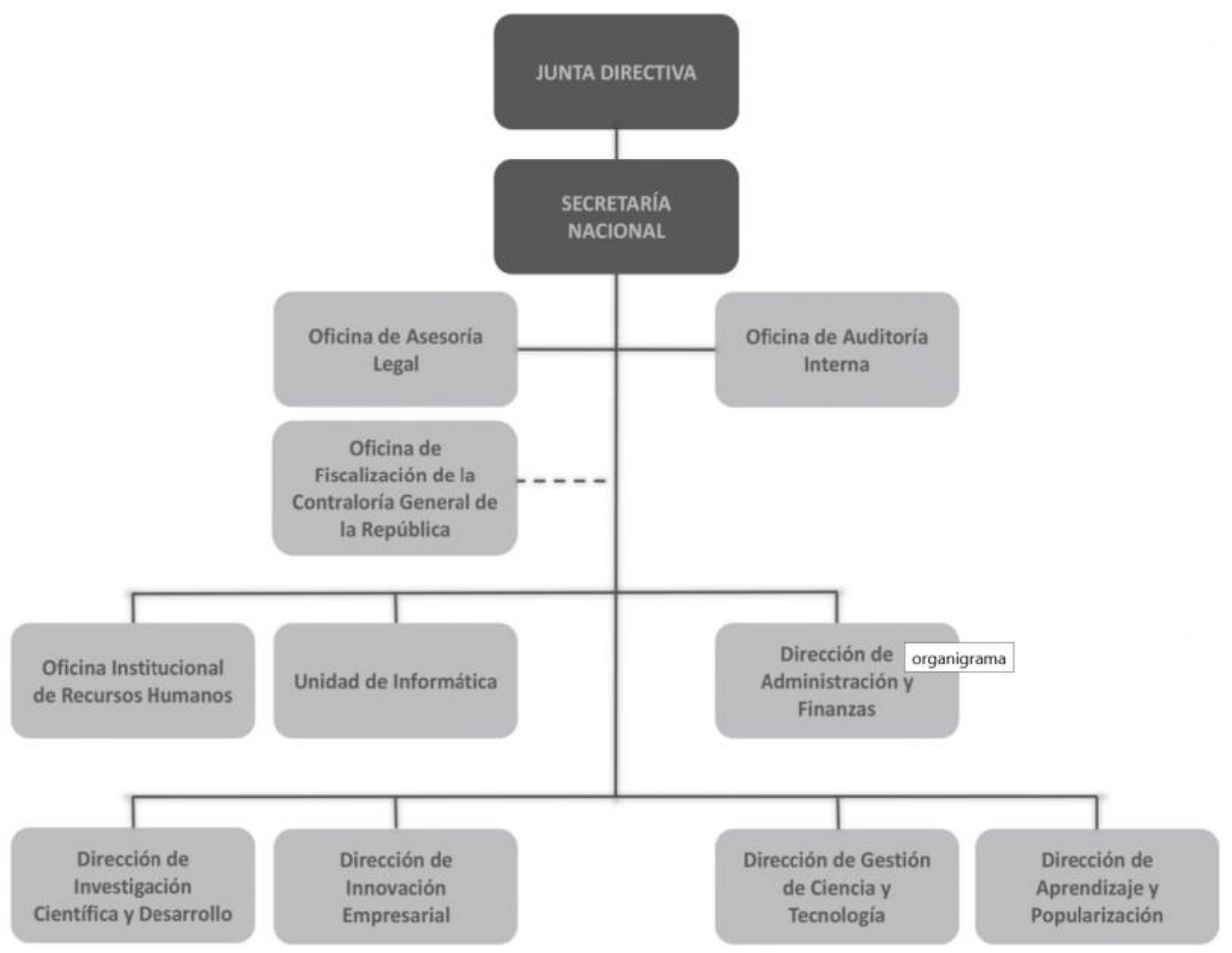

Fuente: http://www.senacyt.gob.pa/organigrama/

Especialmente relevante en el organigrama de la SENACYT son las cuatro direcciones que son las encargadas de gestionar las diferentes acciones que desarrollan: a) Dirección de Investigación Científica y Desarrollo; b) Dirección de Innovación Empresarial; c) Dirección de Gestión de Ciencia y Tecnología y d) Dirección de Aprendizaje y Popularización

Tal y como se ha señalado con anterioridad, la SENACYT marca su actuación en el marco de la Política Nacional de Ciencia, Tecnología e Innovación de Panamá y Plan Nacional (2015- 
2019) (Panamá, 2015), documento editado por la Secretaría Nacional de Ciencia, Tecnología e Innovación (SENACYT), y del cual extraemos los principales objetivos.

Antes de enumerarlos, es importante la declaración de intenciones que, de manera sucinta, se lleva a cabo en el resumen ejecutivo de dicho documento, y que se resume en los siguientes elementos (Panamá, 2015):

"La ciencia, la investigación, el desarrollo tecnológico y la innovación (CIDTI) son hoy los principales conductores del crecimiento económico y la competitividad, del desarrollo social y de la cultura. Panamá no tiene posibilidad alguna de continuar exitosamente su actual camino de progreso sin capacidades construidas en ellas", por lo tanto, se le otorga un valor estratégico en el desarrollo del país que después no tiene correspondencia con alguno de los elementos fundamentales y claves para el desarrollo de una correcta política científica por parte de los poderes público, como sería el aporte económico total con respecto al Productor Interior Bruto del país, que se le otorga desde el Gobierno de la nación.

Además, aporta el documento citado con anterioridad, los grandes desafíos a los que se enfrenta el país, los cuáles son: “1) alcanzar un mayor grado de desarrollo sostenible; 2) avanzar en el desarrollo inclusivo; 3) consolidar altos niveles de competitividad sostenible; y 4) desarrollar una mayor capacidad de generación, adaptación, difusión y utilización de conocimiento". Todos elementos fundamentales para el desarrollo de una nación, sólo accesibles desde la generación de conocimiento, y especialmente de nuevo conocimiento, adaptado a las necesidades y realidad del entorno concreto.

$Y$ de esas necesidades se plasma la política nacional, marcada para los próximos 25 años. Un plan y una visión muy ambicioso que, adelantando conclusiones, le falta apuesta de índole institucional, tanto en recursos humanos (talento humano) como en el importante factor económico. "Debido a que estos son procesos dinámicos y complejos, que incluyen la interacción entre diferentes agentes económicos, académicos, de investigación, empresariales, financieros y otros, que trabajan guiados por incentivos de mercado o de producción de nuevo conocimiento, se hace imprescindible la adopción de un enfoque sistémico para la definición y evaluación de políticas públicas. Este sistema se denomina el Sistema Nacional de Ciencia, Tecnología e Innovación (SNCTI)" (Panamá, 2015).

En el marco de este documento -sin lugar a duda, importante y autocrítico con el Sistema Nacional de Ciencia, Tecnología e Innovación- se indica que han sido muchos los logros pero aún mucho el camino por recorrer. El principal problema sigue siendo la falta de inversión en investigación, ya que en relación con el PIB Panamá rebajó la inversión de 0.4\% en 1990, al 0.2\% en 2012 , siendo la media de la región del $0.7 \%$ y muy especialmente el limitadísimo número 
de grupos de investigación y por lo tanto de investigadores (142,4 por millón de habitantes) una de las tasas más baja del entorno.

La incorporación del sector privado a la investigación es realmente escasa, siendo aproximadamente un $0.2 \%$ del total del gasto, siendo infructuosas las políticas que desde el Gobierno han intentado incentivar la participación.

Por lo tanto, los objetivos marcados en materia de investigación, tecnología e innovación para los próximos 25 años son (Panamá, 2015):

Objetivo Principal 1: "Utilizar la ciencia, la investigación, la innovación y la tecnología para contribuir a afrontar los desafíos del desarrollo sostenible, la inclusión social y el desarrollo de la innovación para la competitividad.

Objetivo Principal 2: Fortalecer el Sistema Nacional de Ciencia, Tecnología e Innovación (SNCTI) a través de: el desarrollo de la ciencia y de la capacidad científica nacional; la profundización de las competencias construidas y aprovechamiento de las ventajas competitivas existentes en el país; el desarrollo del sistema de financiación para la ciencia, la investigación y la innovación; y el reforzamiento de la capacidad de gobernanza del sistema".

Estos objetivos se estructuran en torno a cinco grandes proyectos:

1. Programa “Ciencia, Investigación, Desarrollo Tecnológico e Innovación para el Desarrollo Sostenible". Se pretende generar sinergias para el análisis sobre los problemas del desarrollo sostenible, adaptándose a los cambios, comportamiento y problemas sociales. Para ello, se potencia proyectos y estudios sobre el sistema actual de producción, así como la relación con los recursos hídricos y del suelo, con el fin de garantizar la seguridad alimentaria. También se orienta a implementar políticas energéticas a largo plazo, con la finalidad de luchar contra el cambio climático y un desarrollo urbano sostenible.

2. Programa "Ciencia, Investigación, Desarrollo Tecnológico e Innovación para la Inclusión Social". Programa centrado en la mejora de la educación y la salud, trabajando en aspectos sobre "equidad y calidad" educativa, centrándose en las relacionadas con la competencias de ciencia, tecnología e innovación. También se busca el desarrollo comunitario, siendo la tecnología y la educación el motor para la participación y la integración social, especialmente entre los grupos más desfavorecidos.

3. Programa "Desarrollo de la Innovación Empresarial y el Emprendimiento Dinámico para la Competitividad Sostenible". Dirigido a generar en las empresas la cultura de la competitividad por medio de la innovación y el emprendimiento.

4. Programa "Fortalecer la Ciencia y la Capacidad Científica Nacional". En este caso se centra en la capacitación de capital humano en el marco del sistema universitario, para dirigirlo y centrarlos en el ámbito de la investigación. Para ello, se busca un ámbito universitario con mayor calidad y competitividad, en el cual se desarrolle la investigación. Para ello se quiere incentivar el cambio de modelo de las universidades tradicionales, de aquellas que sólo están orientadas a la enseñanza por aquellas encaminadas a llevar a cabo desarrollos de investigación de calidad. Se intenta también que con este programa se lleve a cabo relaciones y transferencia de conocimiento entre universidad y empresas. También se identifica que se deberán llevar a cabo, por medio de estos programas, de cuantas acciones se estimen oportunas para la mejora de la visibilidad e internacionalización de los resultados de investigación. 
5. Programa "Fortalecer la Capacidad de Gobernanza del Sistema". Programa centrado en la mejora de la gobernanza, teniendo como visión la inversión de al menos 0.7 del PIB en investigación y desarrollo tecnológico, siendo el origen de este presupuesto la financiación pública. Señala igualmente que se debe direccionar la partida presupuestaria al 1\% del PIB para el año 2020, con una financiación estable que garantice el sistema de investigación.

El Plan también destaca el conjunto de acciones encaminadas a fortalecer la SENACYT como organismo conductor del SNCTI: mejora de las capacidades de inteligencia competitivas, capacidad de planificación y evaluación de políticas, y la organización de una estructura organizacional e institucional interna, que tenga relación con la ciencia panameña y compatible con las tendencias para la gestión moderna de instituciones públicas.

En la memoria anual de SENACYT, siendo la última disponible la del año 2015, se observa algunos datos que nos aportan lo escaso del alcance de las ayudas. Dicha memoria señala la existencia de un programa de becas internacionales, donde en el año 2015 concedieron un total de 78 becas, 52 para maestrías y 26 para doctorados (SENACYT, 2015). Igualmente el número de proyectos adjudicados para I+D es un número también escaso que oscila (y desaparecen) atendiendo a cuestiones presupuestarias: año 2004, 8 proyectos; año 2005, 41 proyectos, año 2006, 42 proyectos; año 2007, 52 proyectos; año 2008, 46 proyectos; año 2009, 42 proyectos; año 2010, 58 proyectos; año 2011, 48 proyecto; año 2012, 7 proyectos, año 2013, 0 proyectos; año 2014, 0 proyectos; año 2015, 72 proyectos con un total 4.47 millones de dólares (Senacyt, 2015). Se puede observar como durante el tiempo en el que el Gobierno de Panamá destinó cuantiosas cantidades de millones de dólares para la ampliación del Canal, no hubo proyectos aprobados ya que se dejó de convocar dichas ayudas.

Importante también es la percepción de la ciudadanía sobre la ciencia, publicada recientemente en la V Encuesta de Percepción Social de la Ciencia y la Tecnología 2017 (IME, 2017), de la que se extraen las siguientes conclusiones: perciben un futuro bueno para la ciencia, aunque interpretan el retraso actual; la mitad de los panameños estiman que los científicos cuentan con buena infraestructura; el 70\% piensan que se debería invertir más en I+D; se reclama mayor difusión de los contenidos. Salud, religión y deportes, en ese orden, son las cuestiones por las que la población de Panamá se encuentra interesados. Tras éstos estarían los temas científicos, junto con los medioambientales.

\section{Estado de la producción científica panameña}

Medir la ciencia nunca es una tarea simple. La cienciometría está siendo cada vez más utilizada para el análisis y valoración de las publicaciones de un investigador de forma individual, cuando nos deben facilitar tendencias en la producción y análisis de áreas de investigación. La 
bibliometría, generada para determinar qué revistas debían adquirir para sus colecciones los bibliotecarios, está siendo utilizada de forma perversa por muchos estamentos gubernamentales, especialmente para medir la producción científica. Entendemos que las metrias tienen graves problemas y carencias que deben ser solucionadas para mejorar la evaluación individual de los investigadores, pero que sí pueden aportar datos relevantes y fiables para analizar y determinar políticas científicas.

Vamos a utilizar una serie de herramientas para describir el estado de la producción científica panameña, que no es en términos absolutos, la investigación científica del país. No todo lo que se investiga debe acabar en publicación, aunque realmente sería una pérdida que las conclusiones de las investigaciones no fueran difundidas. Otra cuestión es dónde difundirlo, dónde publicarlo. Aunque escépticos y críticos, como hemos dicho anteriormente, con los sistemas de medición de la ciencia basados únicamente en el factor de impacto como elemento clave, sí vamos a utilizar una herramienta privada (con sus intereses comerciales) como es la herramienta del grupo de investigación Scimago, realizada para Scopus.

Con dicha herramienta vamos a llevar a cabo la determinación del posicionamiento de Panamá como potencia de publicación en la región y su posición a nivel mundial, así como cuáles son aquellas áreas en las que se con mayor asiduidad publican sus autores.

Para otras cuestiones planteadas, tales como el número de investigadores e inversión en I+D+i utilizamos la web "Index Mundi" la cual recoge datos de agencias solventes, como se describirá en cada uno de sus gráficos.

Piedra y Martínez (2007) señalan que los investigadores publican los resultados de sus investigaciones por prestigio y desarrollo profesional. Igualmente indican que se lleva a cabo esta labor de difusión como método de divulgación de aquellos elementos descubierto en sus investigaciones, y que éstas publicaciones posicionan a una determinada comunidad científica y a éstos de manera individual. El publicar otorga un reconocimiento (Piedra; Martínez, 2007) que incentiva nueva producción científica.

La fórmula por la que en la actualidad se difunden los trabajos de investigación es el artículo publicado en revistas científicas, ya que éste es el tipo documental con mayor reconocimiento científico, académico y gubernamental (importante a la hora de lograr complementos económicos por la labor investigadora o como método de evaluación para lograr la aceptación de proyectos de investigación). La difusión por medio de artículos es el método rápido (cada vez menos, ya que el sistema de publicación está prácticamente colapsado por los requerimientos de publicar en revistas de impacto) por excelencia junto con el acta de los congresos, por el cual muchos investigadores apuestan por su uso. 
Por ello, y para contextualizar y mostrar la producción científica general de los científicos e investigadores panameños hemos usado la herramienta Scimago Journal \& Country Ranking, las cuáles facilitan una serie de opciones que posibilitan comparar y analizar la producción científica de un país.

En la imagen 2 se ofrece un cuadro general con la producción total de trabajos indexados en esta herramienta. En este caso hemos realizado la consulta para todo tipo de temáticas y trabajos, acotando sencillamente por la ubicación geográfica (América Latina). Panamá se encuentra en el puesto 13, por debajo de Costa Rica y por encima de Trinidad y Tobago, con un total de 5712 trabajos. Por lo tanto, Panamá está situada en el puesto 13 de 48.

Imagen 2: Producción científica total en Scopus. Filtro: América Latina

\begin{tabular}{|c|c|c|c|c|c|c|c|c|}
\hline \multicolumn{3}{|c|}{ Country } & \multirow{2}{*}{$\begin{array}{r}\downarrow \text { Documents } \\
749498\end{array}$} & \multirow{2}{*}{$\begin{array}{r}\text { Citable documents } \\
715170\end{array}$} & \multirow{2}{*}{$\begin{array}{l}\text { Citations } \\
7557916\end{array}$} & \multirow{2}{*}{$\begin{array}{r}\text { Self-Citations } \\
2501838\end{array}$} & \multirow{2}{*}{$\begin{array}{r}\text { Citations per Document } \\
10.08\end{array}$} & \multirow{2}{*}{$\begin{array}{r}H \text { inde, } \\
46\end{array}$} \\
\hline 1 & Q & Brazil & & & & & & \\
\hline 2 & [י] & Mexico & 258077 & 245164 & 2849413 & 568014 & 11.04 & 352 \\
\hline 3 & $=$ & Argentina & 174968 & 165384 & 2389147 & 480251 & 13.65 & 337 \\
\hline 4 & 늘 & Chile & 116283 & 110779 & 1525554 & 286939 & 13.12 & 291 \\
\hline 5 & $=$ & Colombia & 71966 & 68319 & 629134 & 93289 & 8.74 & 216 \\
\hline 6 & $\approx$ & Venezuela & 35778 & 34327 & 381824 & 45129 & 10.67 & 181 \\
\hline 7 & $\equiv$ & Cuba & 33861 & 32388 & 245917 & 43708 & 7.26 & 145 \\
\hline 8 & D & Peru & 17096 & 15581 & 254779 & 26504 & 14.90 & 179 \\
\hline 9 & 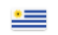 & Uruguay & 15428 & 14591 & 235388 & 30044 & 75.26 & 150 \\
\hline 10 & $\equiv$ & Puerto Rico & 14773 & 14150 & 300572 & 17779 & 20.35 & 184 \\
\hline 11 & 풀 & Ecuador & 10486 & 9865 & 127477 & 15270 & 12.16 & 126 \\
\hline 12 & $\equiv$ & Costa Rica & 10248 & 9611 & 184108 & 18130 & 17.97 & 152 \\
\hline 13 & 룰 & Panama & 5712 & 5369 & 175231 & 15566 & 30.68 & 162 \\
\hline 14 & $\Delta$ & Trinidad and Tobago & 5509 & 4971 & 54188 & 3885 & 9.84 & 84 \\
\hline 15 & $\infty$ & Jamaica & 5296 & 4651 & 61611 & 5771 & 11.63 & 83 \\
\hline 16 & 0 & Bolivia & 3939 & 3730 & 79603 & 5898 & 20.21 & 103 \\
\hline 17 & 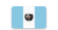 & Guatemala & 2582 & 2369 & 37322 & 2339 & 14.45 & 78 \\
\hline
\end{tabular}

Fuente: http://www.scimagojr.com/countryrank.php?region=Latin\%20America

Datos obtenidos el 13 de febrero de 2018 
Pueden observar como destaca claramente Brasil con 747.498, seguido por México y Argentina con un total de 258.077 y 174.968 documentos indexados respectivamente. Con posterioridad se observa una bajada importante, siendo menor a los 20.000 documentos publicados por debajo de la posición ocupada por Cuba (posición 7).

La posición de Panamá (13 de 48 en América Latina) no parece muy alta, pero sí que puede entenderse como importante si lo ponemos en relación con el número de investigadores (muy escaso) y con el número de publicaciones propias que aparecen en Scopus. Esta cuestión es relevante ya que no existe una sola revista panameña indexada en la base de datos Scopus.

Si la comparativa la hiciéramos a nivel mundial, Panamá caería hasta la posición 104 de 239 países.

Imagen 3: Revistas en las que publican los autores con nacionalidad panameña

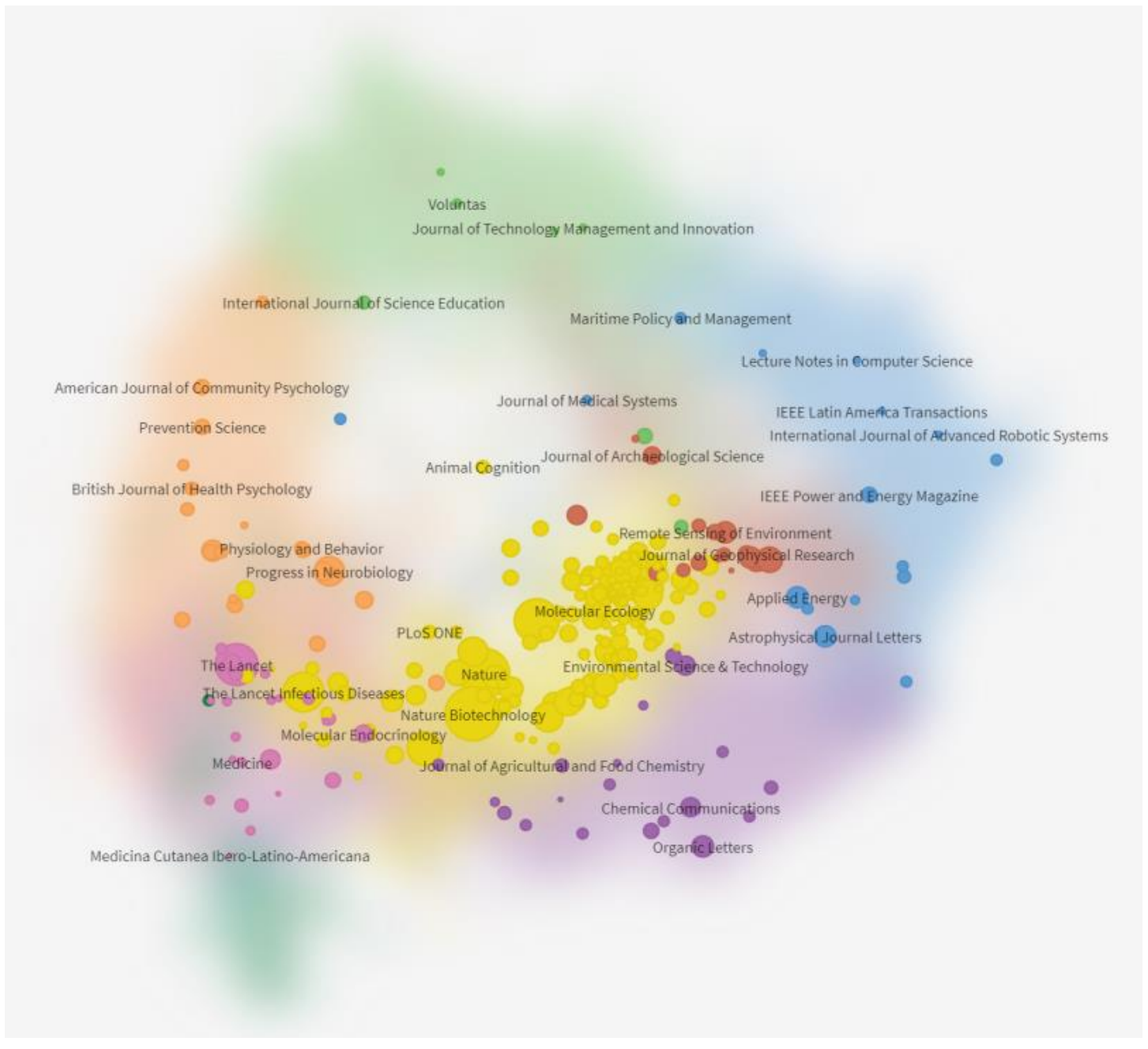

Fuente: http://www.scimagojr.com/shapeofscience/ 
En la imagen 3 pueden observar las revistas en las que están presentes autores de nacionalidad panameña, destacando "Nature Biotechnology", "The Lancet", "Science", "Nature", entre otras. Debe quedar claro que no tiene por qué ser publicaciones en coautoría simple, pudiendo ser firmados por más de un autor de diferentes nacionalidades.

En la imagen 4 se aportan el mapa de publicaciones por áreas. Se puede observar cómo se destaca en las áreas de "Agricultural and Biological", "Earth and Planetary Sciences", "Physics and Astronomy", "Medicine", etc. Las áreas de las Ciencias Sociales y las Humanidades están muy poco representadas en la producción científica panameña.

Imagen 4 Mapa de publicaciones por áreas

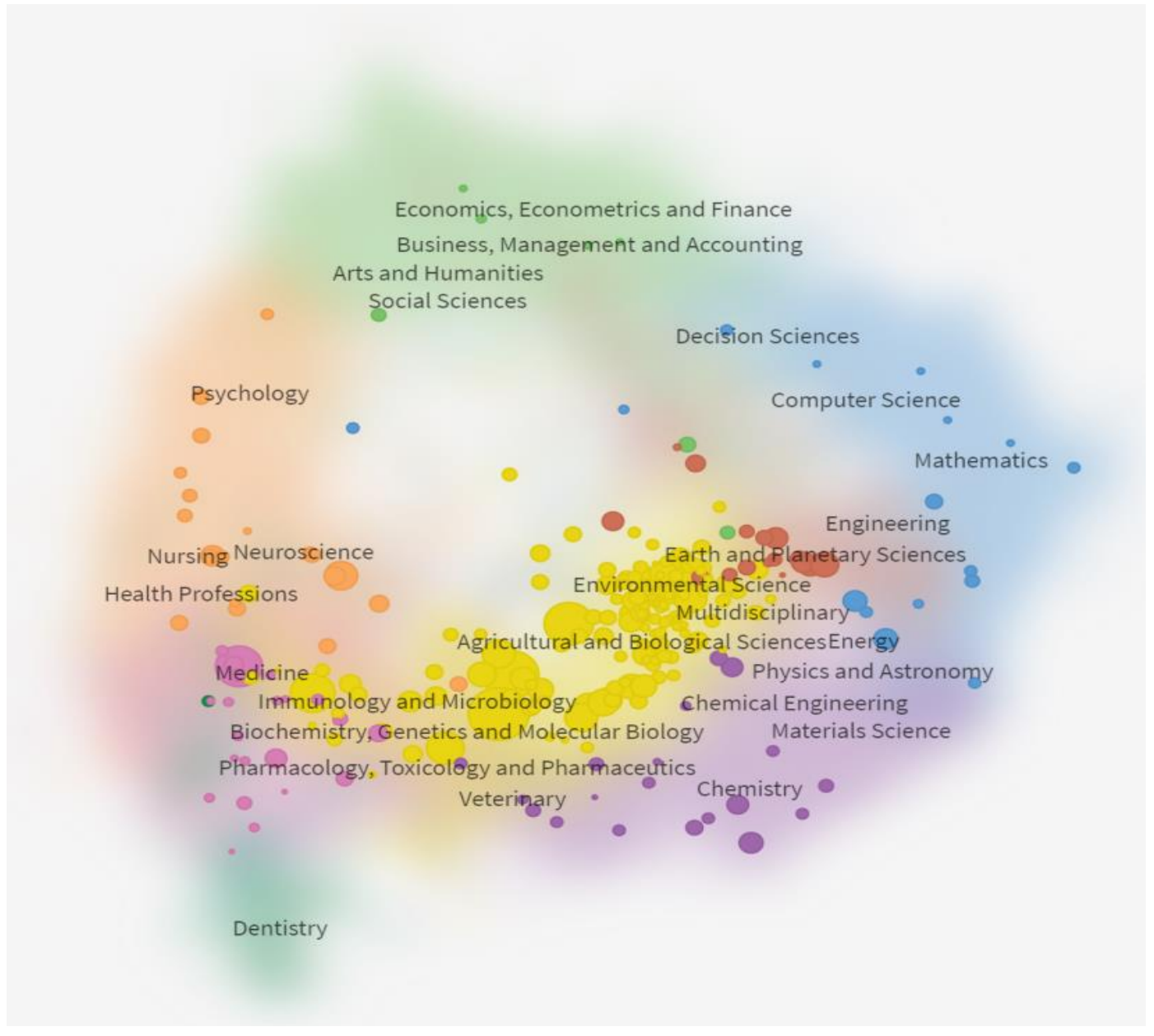

Fuente: http://www.scimagojr.com/shapeofscience/

Un elemento fundamental en cualquier política científica que ya ha sido tratada, pero no por ello no deba incidirse, es la inversión en I+D. La web de Index Mundi (https://www.indexmundi.com/es) facilita la posibilidad de análisis por dichos criterios, como 
por otros igualmente relevante. La imagen 5, destacada para América Central y el Caribe, otorga a Panamá una inversión del 0,2\% del PIB (Fuente: Instituto de Estadística de la Organización de las Naciones Unidas para la Educación, la Ciencia y la Cultura (UNESCO).

Imagen 5: Inversión en I+D (\% PIB). América Latina y El Caribe. Datos del 2010
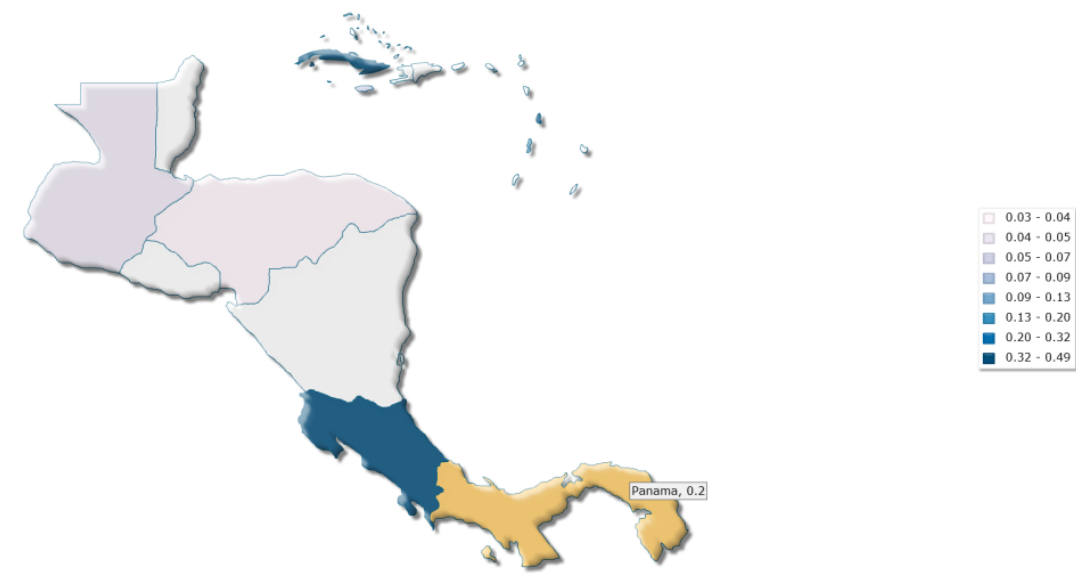

Fuente:

https://www.indexmundi.com/es/datos/indicadores/GB.XPD.RSDV.GD.ZS/map/centralamerica

Con respecto al número de investigadores Panamá se nos presente con un pobre 111,47 por cada millón de habitantes, lo que supone no más de 500 investigadores en todo el país, número muy escaso si se quiere conseguir una producción científica con una cantidad tan significativa como para poder comenzar a tratar de la calidad de la producción.

Imagen 6. Investigadores en I+D. Datos del 2010 


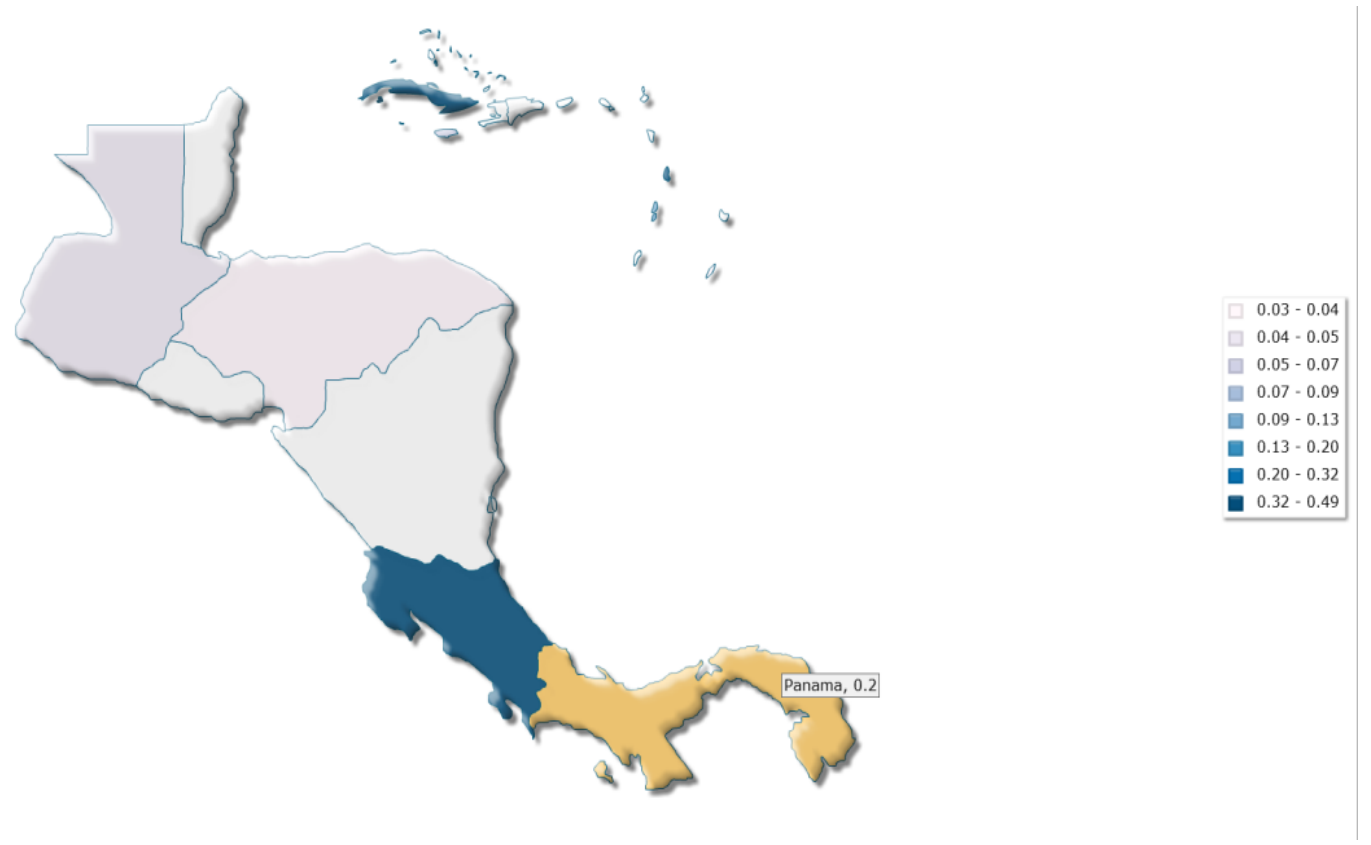

https://www.indexmundi.com/es/datos/indicadores/SP.POP.SCIE.RD.P6

Hemos intentado aportar lo que estimamos que son los principales datos globales que nos aporta una macro visión general de la situación de la inversión y producción científica tras el esfuerzo de I+D+i de Panamá. Evidentemente pueden tenerse en cuenta otra serie de parámetros, e incluso otras fuentes pueden facilitarnos datos distintos. No obstante, las fuentes utilizadas son solventes aunque en algunos de los casos los datos no están especialmente actualizados. No obstante, no difieren especialmente en los que aporta la SENACYT por medio de sus documentos publicados.

\section{Discusión y líneas de actuación}

La SENACYT especifica una serie de áreas de actuación en materia de I+D. Como veremos con posterioridad alguno de ellos no acaba de comenzar su andadura de manera eficiente, no obstante se indican a continuación con el fin de plasmar los intentos importantes por parte de la institución. Las áreas son las siguientes: a) Financiamiento de proyectos de investigación científica a través de convocatorias públicas; b) Apoyo a la dotación de infraestructura y equipamiento científico; c) Apoyo a la inserción de ex becarios de programas de doctorados de investigación; d) Fortalecimiento de la carrera del investigador científico, con un modelo de reconocimiento de la excelencia, a través del Sistema Nacional de Investigación SIN; e) Facilitar el acceso a bases bibliográficas científicas especializadas, a través de la plataforma ABC; f) Generación de capacidades nacionales a través de talleres de redacción y escritura científica; $g$ ) Propiciar espacios de interacción e intercambio entre comunidad científica, comunidad 
académica y sociedad en general, y h) Apoyo a la generación de Patentes Nacionales (SENACYT, 2017).

Para llevar a cabo estas acciones se han desarrollado tareas tales como la publicación de ofertas para la ciudadanía en general y para los investigadores en especial, publicando convocatorias para la concesión de becas, incentivos laborales y económicos para investigadores destacados, apoyo a proyectos de I+D tanto para centros de investigación como para la empresa privada, incentivar la colaboración internacional, recuperación de recursos humanos del extranjero con alto valor estratégico, todas las anteriores por medio de concurso público y competitivo.

Todos estos procesos son evaluados por pares, siendo en muchos casos los evaluadores destacados investigadores de otros países. Dicha evaluación se suele combinar con evaluación virtual para las primeras fases del proceso con evaluación presencial para la toma de decisión por medio del análisis y discusión de los proyectos.

Vamos a separar las posibles líneas de actuación en materia de I+D+i, y por la tanto la implementación de una política científica nacional, atendiendo a líneas directas orientadas para las empresas y otra línea diferentes para las instituciones tradicionales de investigación, tales como universidades e institutos de investigación. Esta división clásica en la financiación y ayuda a la investigación viene motivada por las formas y fines tan diferentes de cada uno de estos grupos, ya que mientras los primeros van a buscar investigación asociado a su actividad económica y con rendimientos económicos claros, las universidades e institutos de investigación tienen otra finalidad bien distinta, especialmente si son organismos públicos. Tanto es así que durante una época se hacía una clara diferenciación entre las investigaciones aplicadas, presentes en el sector privado, y las investigaciones básicas realizadas especialmente por universidad y organismos públicos estatales. No obstante, esta diferenciación se ha diluido con el tiempo, derivado de que las instituciones financiadoras requieren y financian cada vez menos la investigación básica y centran los esfuerzos en la aplicada, especialmente si puede tener transferencia con los sectores productivos de la sociedad. No vamos a entrar a valorar si la separación inicial era la ideal o si la tendencia actual es la más correcta, solo incidir en que estimamos que ninguna de las dos opciones es la correcta. Actualmente la investigación está tan orientada al mercado que en ciertas ocasiones no se aprecia la apremiante necesidad de hacer investigación sosegada, básica y no con aplicación a corto o medio plazo.

\section{I+D+i para empresas.}

Hacer la distinción propuesta no siempre será fácil ni efectiva. Sobre todo por la escasa investigación e inversión en investigación por parte de las empresas panameñas, de ahí que sea 
necesario la creación y generación de sinergias entre las instituciones públicas y privadas, empresariales y universitarias, investigadoras y productivas.

Acción 1. Convocatoria competitiva para Proyectos de I+D+i.

Se debe llevar a cabo convocatorias competitivas específicas para empresas, en las cuáles se tenga presente la necesidad de financiación tanto por parte de los organismos públicos como por la propia empresa. Igualmente debe quedar claro cuál es la parte de corresponsabilidad social que tendrá la empresa con los hallazgos, es decir, debe quedar explicitado en qué se va a beneficiar la sociedad panameña del proyecto de investigación que cuenta con recursos públicos. No debe financiarse proyectos empresariales que sólo tengan en cuenta el rendimiento neto y beneficio de las empresas. Estas convocatorias deben ser regulares y estables, de tal manera que las empresas conozcan de su publicación y regularidad.

Acción 2. Convocatoria competitiva para Proyectos de I+D+i Universidad-Empresa.

Al igual que en la anterior acción, se deberá contar con convocatorias regulares, en las que se potencie y se beneficie la relación entre universidad y empresa privada. Aunque puede ir encaminada a la investigación aplicada y a la consecución de la mejora de resultados empresariales, esta labor deberá tener una transferencia de resultados hacia la sociedad.

Acción 3. Integración temporal Talento Humano a empresas.

La contratación y/o integración de talento externo (nacional o extranjero) a la empresa puede ser un elemento motivador y ciertamente innovador para la mejora de los procesos de producción e incluso investigador para algunas empresas panameñas. Esta captación de talento para empresas debe ser sufragada por ambas partes, por lo que el coste debe estar muy justificado por parte de las empresas postulantes. La integración de un talento externo debe estar justificada, al igual que se debe conocer las mejoras que se prevén con la integración en el equipo de trabajo. Dicha integración siempre será de carácter temporal y con unos objetivos y temporalización predeterminados.

Acción 4. Fomento de creación de "semilleros de empresas".

Esta ayuda está orientada a la creación de nuevas empresas, especialmente en el ámbito de la pequeña y mediana empresa. Para ello se crearán estructuras públicas estatales para la integración de estos nuevos equipos de trabajos empresariales en estructuras fijas, creando sinergias con otras empresas dentro del mismo semillero. Pensado y orientado para jóvenes empresarios que necesitan una pequeña ayuda para comenzar su andadura, especialmente en aspectos relacionados con la parte de gestión administrativa así como recursos inmuebles.

Acción 5. Creación de oficina gubernamental para la generación de patentes.

Potenciación, asesoramiento y financiación de los procesos de generación y registro de patentes, generadas tanto por la investigación financiada con fondos públicos, como la realizada 
por empresas privadas (financiadas o no con proyectos competitivos). Oficina integral de ayuda al registro de patentes y todo lo derivado de su gestión.

\section{I+D+i para universidad y centros de investigación.}

Sin duda, el principal esfuerzo ya que es el principal impulsor de la Ciencia, deben ser las instituciones universitarias así como los centros de investigación, tanto aquellos que son de carácter público como los privados. Incluso, es sin duda interesante, la potenciación de las relaciones entre ambas realidades, muy presentes en la República de Panamá. El número total de acciones van tanto encaminadas a la generación de la investigación y desarrollo como al control de la calidad universitaria, que algunas tendrán relación con la mejora del ámbito de la docencia, pero otras cuestiones son apropiadas para la mejora de los recursos humanos para la generación de conocimiento científico por medio de la investigación.

Acción 1. Promover las estancias de investigadores extranjeros en centros panameños. Importante acción para la recepción de talento humano extranjero y repatriación del talento nacional. Siguiendo la línea del proyecto Prometeo realizado en la República del Ecuador, estos investigadores se incorporarían a las estructuras estables de las universidades especialmente con fines de investigación, asesoramiento, mejora de equipos humanos, etc., no obstante, también puede estimarse relevante su aportación como docentes en maestrías y para la dirección de doctorandos nacionales (PhD).

La incorporación debería ser siempre superior a los seis meses, y la calidad científica de los postulantes debería estar garantizada por una evaluación rigurosa de los méritos. Igualmente el postulante debe estar avalado por al menos una institución investigadora, que será a la cual se incorporará para realizar las labores de investigación. Se podrían incorporar investigadores al sistema nacional de ciencia y tecnología en caso de que el estado contase con algún programa determinado que implementar, de lo contrario, sería interesante que estos investigadores siempre estuvieran asociados a un centro universitario o a un instituto de investigación.

Es relevante la mejora en la investigación que puede acarrear esta acción, pero especialmente potenciará la internacionalización y visibilidad de los trabajos de investigación. No es desdeñable en absoluto la mejora que puede conllevar a los alumnos de maestrías y doctorados, que contarán con la posibilidad de llevar a cabo investigaciones tanto en el país como en el exterior, por medio de los contactos y convenios que se realicen utilizando a estos investigadores como transmisores.

Acción 2. Promover el envío de talento nacional a formarse en maestrías y doctorados en universidades extranjeras de alta capacitación. 
Esta acción está encaminada a becar a alumnos de maestrías y doctorandos, como método de mejora de su formación y como elemento de mejora de sus dotes investigadores. Además, este intercambio de conocimiento también puede lograr por un lado el contar con doctores egresados por universidades extranjeras y el intercambio de métodos y conocimiento científico.

Pero no sólo debe estimarse que las becas a alumnos es la única opción. El envío de miembros del sistema nacional de investigación, en forma de becas postdoctorales, es un buen mecanismo de mejora de la calidad y la cantidad de investigación. Aunque esta acción repercute directamente en un número de personas menor que la Acción 1 (al ser la primera mucho más intrusiva en el entorno académico e investigador del país), sí que el contacto de investigadores panameños con otros científicos ayudará posteriormente al regreso de éste al país con conocimiento novedoso en técnicas, metodología y líneas de trabajo. No obstante, quede claro que la selección en este proceso es muy importante, selección que debe tener una multiplicidad de vías de análisis: validez y calidad del alumno, objetivos marcados y finalidad de la formación/investigación, y la relevancia del centro de investigación e investigadores de acogida.

Acción 3. Creación de Institutos de Investigación de carácter público.

Potenciar y financiar la creación de institutos tanto públicos como privados dedicados a la investigación en temáticas estratégicas para el país. No obstante, dichos Institutos en el caso de que fueran públicos, podrían estar integrados en una institución pública en el cual se albergarán los diferentes grupos de investigación con alto potencial y valor estratégico nacional.

Acción 4. Creación de spin-off de base tecnológica.

Relacionadas con institutos de investigación y universidades, potenciadas y controladas por éstas, se podrían implantar un sistema de financiación de estas empresas que ofrecen servicios a empresas de los diferentes sectores estratégicos del país. Es una forma clara de relacionar el ámbito empresarial con la realidad universitaria, por lo que estas empresas es una acción conducente a la mejora de la producción por medio de la investigación, tanto para las propias empresas panameñas como para el sector universitario, la cual se convierte en generadora de empleo y riqueza.

Acción 5. Canales de distribución de la Ciencia

Es relevante el potenciar canales internos, nacionales, de procesamiento y difusión del conocimiento científico generado por los grupos de investigación. Los cauces habituales siguen siendo las revistas científicas, congresos y publicación de monografías (DORA, 2012). Estas son los métodos que deberían seguir siendo potenciados.

Para la difusión por medio de congresos, señalar la necesidad de ayudar a las instituciones a generar sus propios ámbitos de difusión, aunque pueda ser igualmente relevante 
la inscripción y presentación de investigación en congresos internacionales. Es evidente que los costos pueden ser altos, por lo que deberán ser analizados para valorar la proporción de coste/eficacia antes del envío a un investigador a un Congreso.

Respecto a las revistas, hemos de analizarlo desde una doble vía: el envío de trabajos a revistas con alto impacto, y la potenciación de revistas de calidad en Panamá. Para potenciar el envío, se debe garantizar ya sea por la ayuda a grupos de investigación (montante mínimo anual) o por medio de métodos competitivos, la financiación suficiente para realizar tareas tales como llevar a cabo las traducciones -si fuese oportuno- así como los pagos por publicación -si es obligado- para la difusión en revistas presentes en estándares internacionales.

Debe formarse a los editores y directores de las revistas científicas panameñas, para inculcarles una cultura de la calidad y de metodología sistemática para el control de todo el proceso editorial de información científica. Todo ello encaminado a una mejora en la calidad y por ende de la visibilidad internacional.

Igualmente, y en el ámbito de la SENACYT, debiera potenciarse una editora propia para actas de congresos como para resultados de investigación en forma de monografías, evidentemente realizando para ello una evaluación por pares para garantizar la calidad del material publicado.

También debe plantearse la creación de un Repositorio nacional de publicaciones del Sistema Nacional de Investigación, es decir, en el camino a la transparencia especialmente de todo aquello publicado por medio de financiación y/o ayudas públicas, se debería garantizar que todo aquello generado por el sistema nacional fuera conocido por la comunidad científica, de tal manera que se garantizase el acceso en libre y gratuito para estos contenidos. La cesión de dichos contenidos debería ser obligatoria para investigadores de instituciones públicas (universidades e institutos) ya que la financiación en pública, así como todos aquellos trabajos producidos al amparo de proyectos, acciones, etc. financiadas por el erario público.

Acción 6. Creación de registro de grupos de investigación

La creación y registro de un catálogo de grupos de investigadores es fundamental para controlar el sistema nacional. Saber quién y qué se está haciendo requiere de un esfuerzo institucional mínimo, pero que podría ser una buena fórmula para encaminar algunas de las acciones propuestas. Por medio de estos grupos, se podría controlar y garantizar una financiación mínima, atendiendo a criterios de producción científica y transferencia de resultados a la sociedad, así como ayudar a la publicación. Igualmente podría servir para, desde aquí, ayudar a la implementación de congresos de calidad, publicaciones con visibilidad internacionales y ayudar en el proceso de la generación de patentes.

Acción 7. Creación de Instituto/Agencia de evaluación de la ciencia en Panamá 
Aunque este organismo puede ser muy complejo de estructurar, es necesario el poder determinar el nivel y calidad de la ciencia y la educación en Panamá.

Para ello, sería necesario el analizar la calidad de los grados universidades, maestrías y programas de doctorados, atendiendo a la temática, profesorado y alianzas internacionales que se tuviera. Igualmente relevante es el análisis y valoración de las universidades existentes, de tal manera que se pudiera conocer cuáles son las universidades más importantes y prestigiosas a tenor de una serie de criterios internaciones, estandarizados y objetivos, de tal manera que puedan ser premiados aquellos centros universitarios con alta capacitación, y en cierta manera, fiscalizar aquellas en las que puedan detectarse graves carencias formativas.

Igualmente, sería desde esta agencia, desde la cual se controlaría el Sistema Nacional de Investigadores y Profesores universitarios, con el fin de que éstos cumplan unos requisitos mínimos previos a la incorporación al sistema universitario o investigador. Además, serviría para valoraciones posteriores, analizando la carrera profesional de los miembros del sistema nacional de investigación.

\section{Conclusiones}

Aunque son innegables los logros que se han alcanzado desde el año 1988, y que se ha desarrollado a través de cuatro planes nacionales, el gasto total del Gobierno con relación al PIB es realmente bajo: del 0,4\% en 1990 , al $0.2 \%$ en 2012 . No vamos a determinar que la media de la OCDE está en el 2,4\% del PIB y que por lo tanto está muy lejos de la actual situación en la República de Panamá, sino que también está baja en comparación con los niveles más altos de la región de países similares, con Costa Rica $(0,5 \%$ del PIB) y Uruguay ( $0.4 \%$ del PIB), datos todos alarmantemente bajos. No se puede llevar a cabo políticas científicas en un país si no existe una financiación clara y una apuesta decidida por la misma.

Dicha cuantía es extremadamente baja, por lo que debe garantizarse por ley una financiación mínima para el sistema de ciencia y tecnología. No puede desarrollarse un sistema solvente, estable y competitivo, si en el año 2012 se cuenta con un total de 500 investigadores para todo el país, número que debería ser el deseado por cada millón de habitantes. La universidad panameña cuenta con una capacidad de investigación muy débil, con pocas universidades de calidad y mal financiadas y con una nula ayuda por parte del sector privado. Entendemos, y desde aquí apostamos, por una financiación estable y fuerte, con un $2 \%$ como mínimo en inversión en PIB, lo cual otorgará capacidad para que SENACYT, o la institución que estimen oportuno, tenga la capacidad de hacer políticas decididas, reales y creíbles, con carácter competitivos y con capacidad de ser difundidas en canales con visibilidad y de carácter internacional. 
La Educación debe ser el motor de todo el sistema, tanto desde donde se genera el nuevo conocimiento como donde deben recaer sus beneficios. Todo el sistema nacional de investigación y transferencia de resultados debe ser una gran espiral en la que el sistema educativo debe estar incluido en todo momento, y debe ser tanto el principio, destinatario y fin del mismo.

Promover desde la Universidad y de los Institutos/Centros de investigación tanto la investigación básica como la aplicada, investigación que debe tener relacionales locales e internacionales (regionales y mundiales), con sinergias de colaboración e investigación a nivel internacional.

Tal y como señala la propia SENACYT existen insuficientes recursos humanos de alto nivel, y sin embargo el plan "Desde Panamá al mundo" no está implementado. Son innegables los resultados por el programa PROMETEO en la República del Ecuador, donde se han incorporado un importante número de investigadores extranjeros que han ayudado a la mejora del sistema nacional, y ha aumentado el número de publicaciones y la visibilidad de los resultados a nivel internacional.

Por lo tanto, parece igualmente necesario el incentivar la producción científica y la difusión de los contenidos en fuentes solventes, tanto de carácter local como internacional.

\section{Referencias}

CRESPI, Gustavo; SOLÍS, Galileo; TACSIR, Ezequiel. Evaluación del impacto de corto plazo de SENACYT en la innovación de las empresas extremeñas. Washington: BID, Banco Interamericano de Desarrollo: División de Ciencia, Tecnología. Sector Social. Notas técnicas IDB-TN-263, abril 2011.

DORA. Declaración de San Francisco de evaluación de la Ciencia: poniendo ciencia en la evaluación de la investigación. 2012. http://blogs.ujaen.es/cienciabuja/wpcontent/uploads/2013/10/dora.pdf Consultado [19/02/2018]

MUNDO SOCIAL. Panamá, el país con mayor crecimiento de América Latina y El Caribe (especial abril, 2017) https://www.mundosocial.net/especiales/panama-pais-mayorcrecimiento-economico-america-latina-caribe/18856 Consultado [19/02/2018]

PANAMÁ. Secretaría Nacional de Ciencia, Tecnología e Innovación. Política Nacional de Ciencia, Tecnología e Innovación de Panamá y Plan Nacional (2015-2019). 2015. ISBN 978-9962-68015-4. 150 p. http://www.senacyt.gob.pa/wp-content/uploads/2017/03/PENCYT2015 2019.pdf

PANAMÁ. Secretaría Nacional de Ciencia y Tecnología (SENACYT). Dirección de investigación científica y desarrollo. 2017. http://www.senacyt.gob.pa/wp-content/uploads/2017/07/10.1Descripci\%c3\%b3n-de-Proyectos-de-ID.pdf Consultado [19/02/2018]

PANAMÁ. Secretaría Nacional de Ciencia y Tecnología (SENACYT). V Encuesta de percepción social de la Ciencia y la Tecnología. 2017. http://www.senacyt.gob.pa/wpcontent/uploads/2017/11/10.3-Encuesta-Percepci\%c3\%b3n-Social-de-la-Ciencia-y-laTecnolog\%c3\%ada.pdf Consultado [19/02/2018] 
PANAMÁ. Secretaría Nacional de Ciencia y Tecnología (SENACYT). Memoria anual. 2015. http://www.senacyt.gob.pa/wp-

content/uploads/transparencia/descargas/memorias/MEMORIA-SENACYT-2015.pdf Consultado [19/02/2018]

PANAMÁ. Secretaría Nacional de Ciencia y Tecnología (SENACYT). Misión, visión y valores. 2018. http://www.educacionsuperior.gob.ec/valores-mision-vision/ Consultado [19/02/2018]

PIEDRA SALOMÓN, Yelina; MARTínEZ RODRÍGUEZ, Ailín. Producción científica. Ciencias de la Información, v. 39, n. 3, p. 33-38, 2007.

WIKIPEDIA. Panamá. 2018. https://es.wikipedia.org/wiki/Panam\%C3\%A1. Consultado $[19 / 02 / 2018]$ 